\title{
Multilayer Phase-Only Diffraction Gratings: Fabrication and Application to EUV Optics
}

Farhad Salmassi, ${ }^{*}$ Eric M. Gullikson, Erik H. Anderson, and Patrick P. Naulleau

Center for X-Ray Optics (CXRO), Lawrence Berkeley National Laboratory, 1 Cyclotron Road, Berkeley, CA 94720, USA

\begin{abstract}
The use of phase-only diffractive devices has long played an important role in advanced optical systems in varying fields. Such devices include gratings, diffractive and holographic optical elements, diffractive lenses, and phase-shift masks for advanced lithography. Extending such devices to the increasingly important regime of extreme ultraviolet (EUV) wavelengths, however, is not trivial. Here, we present an effective fabrication and etch process enabling high-resolution patterning of $\mathrm{Mo} / \mathrm{Si}$ multilayers for use in EUV phase devices, providing another method for fabrication of high numerical aperture diffractive devices or high-resolution EUV phase shift masks

\section{Introduction}

Extending common techniques for the fabrication of optical phase elements to the increasingly important regime of extreme ultraviolet (EUV) wavelengths is anything but trivial. In the past, patterned relief methods providing high efficiency binary gratings have been demonstrated. ${ }^{1}$ These methods suffer from resolution limitations because the deposited multilayer on top of the relief structures cannot grow perfectly conformal to the surface. Inherent rounding effects near feature edges and other deposition-related phenomena $^{2}$ (see Figure 1) make it nearly impossible to precisely translate topography throughout the multilayer stack. In addition to binary gratings, gray-scale e-beam
\end{abstract}


exposure methods capable of high resolution three-dimensional pattering have been used in fabricating both blazed gratings ${ }^{3}$ and EUV diffusers. ${ }^{4,5}$ Similarly, these methods also suffer from the response of the multilayer coating to the underlying resist topography. To address this issue we consider patterning phase-modulating structures on top of or directly into the multilayer. Despite the fact that most materials strongly absorb at EUV wavelengths, molybdenum (Mo) provides an excellent trade-off between absorption and phase shifting. Since silicon ( $\mathrm{Si}$ ) is considered to be virtually transparent at EUV, Mo/Si multilayers provide an excellent means of defining phase shifting features while maintaining the highest efficiency possible.

Using this method of defining phase shifting features and a solid layer of Mo instead of a Mo/Si multilayer, it is possible to fabricate either transmission or reflection devices. This has been implemented in the past on transmission devices, ${ }^{6}$ but suffered from poor efficiency due to problems with etching. The etching of $\mathrm{Mo} / \mathrm{Si}$ multilayer to produce EUV phase shift masks has been proposed and investigated by Han et al. ${ }^{7,8}$ Here we describe a fabrication method, and improved etch processes for high-resolution etching directly into a Mo/Si multilayer stack, using an $\mathrm{Cr}$ etch stop layer which is deposited along with the multilayer deposition. Figure 2 shows an example of 500nmpitch phase shifting multilayer gratings fabricated using this process. Its performance is discussed below.

\section{Theoretical efficiency and design requirements}

The theoretical efficiency of phase gratings defined directly onto Mo/Si multilayers is in principle no different than binary gratings with ideal conformal multilayers. The goal is to achieve $\lambda / 2$ phase shift upon reflection. Here, the phase shifting device is the Mo

\footnotetext{
*Electronic mail: fsalmassi@1bl.gov
} 
embedded within the multilayer. Despite the fact that most materials strongly absorb at EUV wavelengths, Mo provides an excellent trade off between absorption and phase shifting at EUV wavelengths. For a $\pi$ phase shift thickness of $86 \mathrm{~nm}$ at 13.5 -nm wavelength, Mo still transmits $60 \%$ of the power supporting surprisingly efficient phase devices. ${ }^{9}$

Figure 3 (a) and (b) provide some tolerance requirements for grating fabrication. The models show that to maintain an efficiency loss of less than 5\% (relative), the variation from a 0.5 duty cycle needs to be within 0.42 to 0.58 . In addition, to maintain a loss of less than $5 \%$ in relative efficiency, the overall Mo thickness within the $\mathrm{Mo} / \mathrm{Si}$ matrix has to remain between $72 \mathrm{~nm}$ and $91 \mathrm{~nm}$, which appears to be a lenient figure. However, the zero order may have as much as $7 \%$ reflectance if the total Mo thickness is closer to $72 \mathrm{~nm}$ or $91 \mathrm{~nm}$, which is undesirable if the suppression of the zero order is part of the design requirements. Total Mo thickness of $86 \mathrm{~nm}$ corresponds to sixteen $\mathrm{Mo} / \mathrm{Si}$ layer pairs, and ensures total suppression of the zero order.

\section{Fabrication}

As mentioned above, there are great advantages with defining features directly onto $\mathrm{Mo} / \mathrm{Si}$ multilayers, however this presents several fabrication challenges. The primary challenge is developing an etch process that stops precisely below sixteen $\mathrm{Mo} / \mathrm{Si}$ layer pairs. At the $13.4 \mathrm{~nm}$ wavelength, this satisfies the $86 \mathrm{~nm}$ of Mo needed for maximizing the diffracted power into the $1^{\text {st }}$ and $-1^{\text {st }}$ orders and minimizing the $0^{\text {th }}$ order power. To meet this requirement, a chromium $(\mathrm{Cr})$ etch-stop layer was embedded within the multilayer. Cr has extremely high etch selectivity with respect to Si and Mo when using a 
sulfur hexafluoride etch chemistry (details below), and is a good candidate for residuefree pattern transfer. ${ }^{10}$

The next challenge was the fabrication of the multilayer system while controlling the thickness of the embedded $\mathrm{Cr}$ within the multilayer structure. The $\mathrm{Cr}$ etch-stop layer necessarily needs to be exactly one period with respect to the multilayer for maximum efficiency. Deviation from this thickness results in efficiency loss of the entire multilayer system, as shown in Figure 4. Notice that considerably higher theoretical reflectivities are achievable for the multilayer system when the Cr layer is below $2 \mathrm{~nm}$. However, from previous experiments it was observed that Cr thickness $2 \mathrm{~nm}$ does not behave as an adequate etch-stop for the etch chemistry chosen. Thus exactly one period of $\mathrm{Cr}$ is needed to maximize multilayer performance.

When deposited films are relatively thin - on the order of a few nanometers - they form a diffusion layer at their boundaries. This diffusion layer itself is on the order of a few nanometers. Often in thin films this phenomenon results in the contraction of the overall thickness of the film stack. In multilayer systems, the contraction parameter is multiplied by the number of interfaces, and becomes significant. The contraction as a result of interdiffusion of Mo/Si has been well studied and understood. ${ }^{11}$ However, the amount of contraction as a result of interdiffusion of Cr-Mo and Cr-Si is not well known. An empirical approach was used here to deposit an accurate amount of $\mathrm{Cr}$ without the need for accurately understanding $\mathrm{Cr}$ interdiffusion and contraction characteristics. This method is applicable to many other systems.

To find deposition parameters, for exactly one period of $\mathrm{Cr}$ embedded in a $\mathrm{Mo} / \mathrm{Si}$ matrix, we began first by tuning deposition parameters required for fabricating a $\mathrm{Mo} / \mathrm{Si}$ 
multilayer designed to reflect at $13.4 \mathrm{~nm}$. Next, a three-layer system of $\mathrm{Si} / \mathrm{Cr} / \mathrm{Mo}$ was fabricated such that the period would equal exactly twice the Mo/Si system alone, see Figure 5 (a) for detail. X-ray diffraction was used to check the multilayer period. Having fabricated a tri-layer system with twice the desired period ensures the correct deposition parameters needed to embed precisely one period of $\mathrm{Cr}$, anywhere in the multilayer matrix. On the final substrate made for processing, an additional $\mathrm{Cr}$ etch-stop layer was deposited on top of the multilayer stack after reflectivity measurements. This top $\mathrm{Cr}$ layer will act as a hard mask during the initial pattern transfer, which is discussed below. Figure 5 (b) shows a cartoon of the finished multilayer system.

The Mo/Si and $\mathrm{Cr}$ deposition was performed at the CXRO multilayer coatings facility. Mo and Si were deposited using DC magnetron sputtering in argon plasma at a gas pressure of 1.0mTorr with 120watts of DC power for Si, and 80watts of DC power for Mo. The Cr was sputtered using RF power set to 275 watts at the same gas pressure. Deposition was done at a target to substrate distance of 3.5 inches. The deposition system used employs three sputter guns, and thus the entire multilayer stack including etch-stop layer was deposited under one vacuum condition.

In preparation for electron beam lithography and patterning, the substrate was coated with a monolayer of photoresist primer hexamethyldisilazane (HMDS) for good photoresist adhesion. The chemically amplified IBM KRS-XE photoresist of 4.8\% concentration was spin-coated at 2000rpm for 60s. Following resist deposition the substrate was baked on an open hot plate at $120^{\circ} \mathrm{C}$ for 3 minutes resulting in a resist film thickness of approximately $120 \mathrm{~nm}$. Lithography was accomplished using an electron beam tool ${ }^{12}$ at the CXRO nanofabrication facility, operating at $100 \mathrm{kV}$ and $480 \mathrm{pA}$ 
employing an average beam diameter of approximately $7 \mathrm{~nm}$. Two sets of gratings with, $500 \mathrm{~nm}$ pitch, and $1000 \mathrm{~nm}$ pitch at a dose of $35 \mu \mathrm{C} / \mathrm{cm}^{2}$ were exposed. The substrate was then developed in tetramethylammoniumhydroxide (TMAH Shipley LDD-26W) for 60s and rinsed with deionized water.

The next challenge to fabrication was developing an effective etch process. A common chlorine/oxygen chemistry was used to etch through the $\mathrm{Cr}$ hard mask. This was followed by the sulfur hexafluoride $\left(\mathrm{SF}_{6}\right)$ chemistry, which was chosen due to its high selectivity with respect to $\mathrm{Cr}$. Although $\mathrm{SF}_{6}$ provides an effective etch for both Mo and $\mathrm{Si}$, the etch rate for Mo and $\mathrm{Si}$ are not equal. This phenomenon coupled with the quick consumption of KRS resist during etching was the motivating force behind employing the top Cr hard mask.

Etching for pattern transfer was performed at the CXRO nanofabrication facility, on two-chamber Oxford plasma etcher using low-energy inductively coupled $\mathrm{SF}_{6}$ plasma for a residue-free surface relief. $\mathrm{SF}_{6}$ produces minimal residue, while the low DC bias of the plasma minimizes the chances of increasing surface roughness through sputtering of the surface. This is very important in the EUV regime, since any additional roughness, will cause efficiency loss due to scattering. The remaining resist and residual hydrocarbons were then removed by using oxygen descum. Cr etchant was used to remove the Cr hard mask. Figure 6 shows a simplified three-step lithography process. Lastly, the topography was investigated using scanning electron microscopy, and atomic force microscopy. 


\section{Results and discussion}

The results of the grating performance are shown in Figure 7(a). Recall from the model that maximum efficiency for the $1^{\text {st }}$ orders is $40 \%$ relative to the overall multilayer efficiency. For an absolute multilayer reflectance of 55\%, this translates to a maximum theoretical absolute efficiency of $22 \%$. The measured absolute reflectance of the $1^{\text {st }}$ orders is $19.4 \%$ in the worst case. This means that the gratings are better than $88 \%$ efficient with respect to the ideal case. Also note that the positive and negative first orders have virtually equal power. This is one advantage over the example of Figure 1, where the multilayer was deposited directly on top of optical elements. Non-uniform multilayer growth over surface relief structures can cause asymmetry in the order strengths, which can be undesirable.

Reflectometry and efficiency measurements were performed at the CXRO calibration and standards beamline (6.3.2) ${ }^{13}$ at the Advance Light Source (ALS) in Berkeley, CA. The reflectivity of the multilayer before hard mask deposition was $63.7 \%$, which became $54.8 \%$ after processing, see figure 7 (b). This $\sim 9 \%$ drop in reflectivity, as a result of processing, indicates an increase in the surface roughness, which was measured at $1.0 \mathrm{~nm}$ rms on the AFM. This is most likely the result of the hard mask removal process, and solicits further investigation in order to increase absolute efficiency.

\section{Summary}

We have demonstrated a technique for fabricating high efficiency diffractive optical elements directly onto Mo/Si multilayer structures and shown that relative efficiencies of better than $88 \%$ are possible. Extending such techniques can play an important role in 
sophisticated optical systems such as diffractive and holographic optical elements,

diffractive lenses, and phase-shifting masks for advanced lithography.

\section{Acknowledgements}

This work was supported in part by DARPA under Contract No. MDA972-98-C-0007

and by the Director, Office of Science, Office of Basic Energy Sciences, Materials

Science and Engineering Division, U.S. Department of Energy under Contract Nos. DE-

AC03-76SF00098 and W-31-109- ENG-38.

\section{Figure Captions}

Figure 1) SEM micrograph of Mo/Si multilayer deposited with DC magnetron sputtering on top of binary gratings. Sputter plasma having non-uniform radial angular distribution, propagates topography along the average direction of incident flux denoted here by the $\beta$ angle. Also notice the smoothing effect of multilayer as features propagate throughout the multilayer volume.

Figure 2) SEM micrographs of an etched multilayer grating with 500-nm period. Etch depth is set to approximately $115 \mathrm{~nm}$, yielding a $\pi$ phase shift on reflection (round trip). Embedded $\mathrm{Cr}$ etch-stop layer fabricated to exactly one multilayer period ensures suppression of the zeroth order and highest possible performance.

Figure 3) (a) and (b) Idealized model for the efficiency of square wave gratings and the relationship between the zeroth and the first orders.

Figure 4) Theoretical model for the performance of a Mo/Si multilayer with embedded $\mathrm{Cr}$ buried 16 layers below the surface.

Figure 5) (a) Schematic of $\mathrm{Si} / \mathrm{Cr} / \mathrm{Mo}$ trilayer cross-section, used to calibrate $\mathrm{Cr}$ deposition parameters. (b) Schematic of multilayer system, with embedded $\mathrm{Cr}$ etch-stop layer and $\mathrm{Cr}$ hard mask, ready for lithography and pattern transfer.

Figure 6) Simplified three-step lithography process for pattern transfer. (a) KRS-XE ebeam resist used to define features with e-beam lithography. (b) Resist development, plus $\mathrm{Cl} / \mathrm{O}_{2}$ chemistry to etch through $\mathrm{Cr}$ hard mask, and $\mathrm{SF}_{6}$ chemistry to etch through multilayer. (c) Removal of residual hydrocarbons using $\mathrm{O}_{2}$ descum, and $\mathrm{Cr}$ wet etchant to remove Cr hard mask.

Figure 7) Results. (a) Measured grating efficiency, better than $88 \%$ with respect to the ideal. (b) Measured multilayer reflectivity, before and after processing, showing the result of increased surface roughness. 
Figure 1

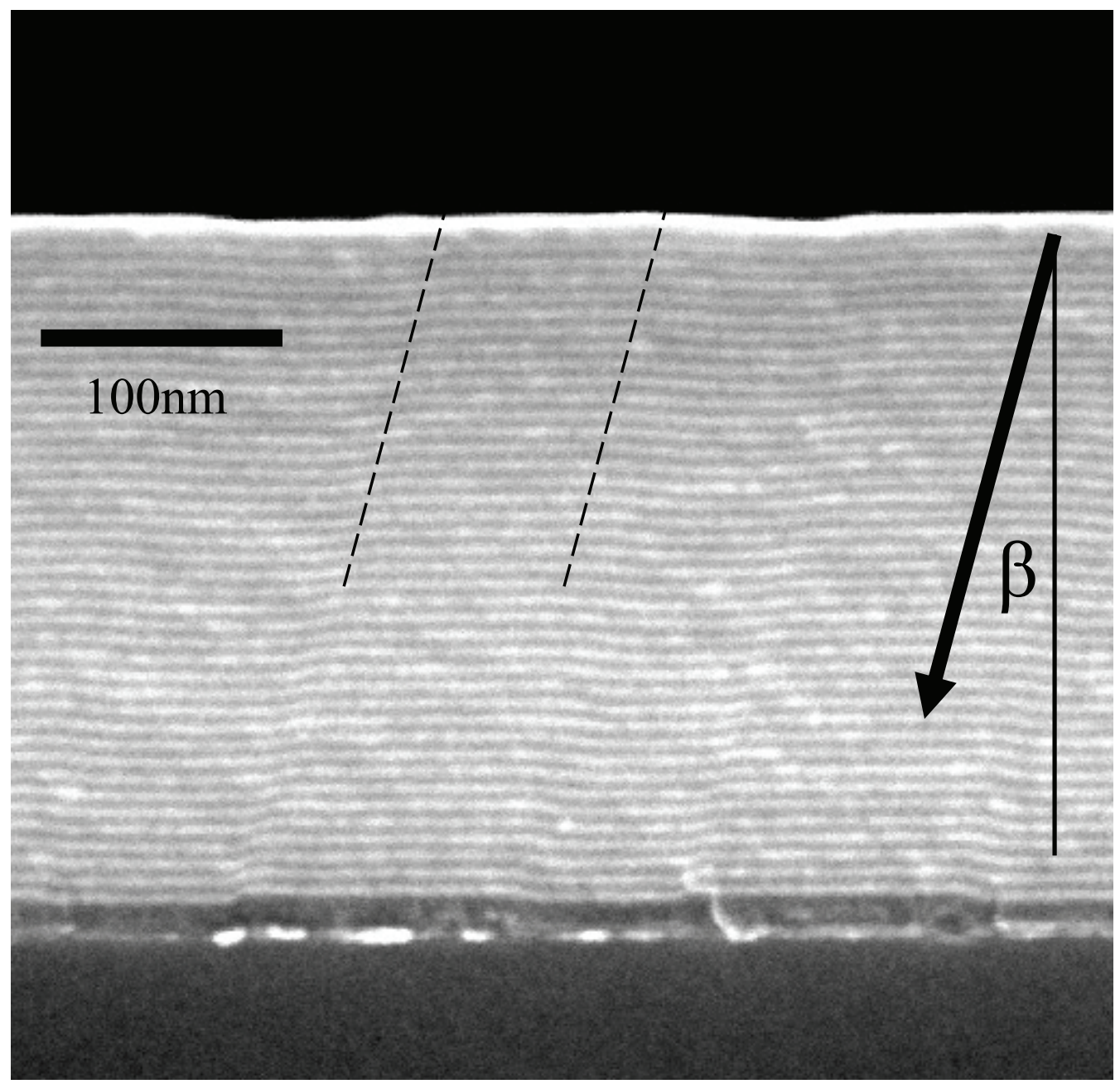


Figure 2

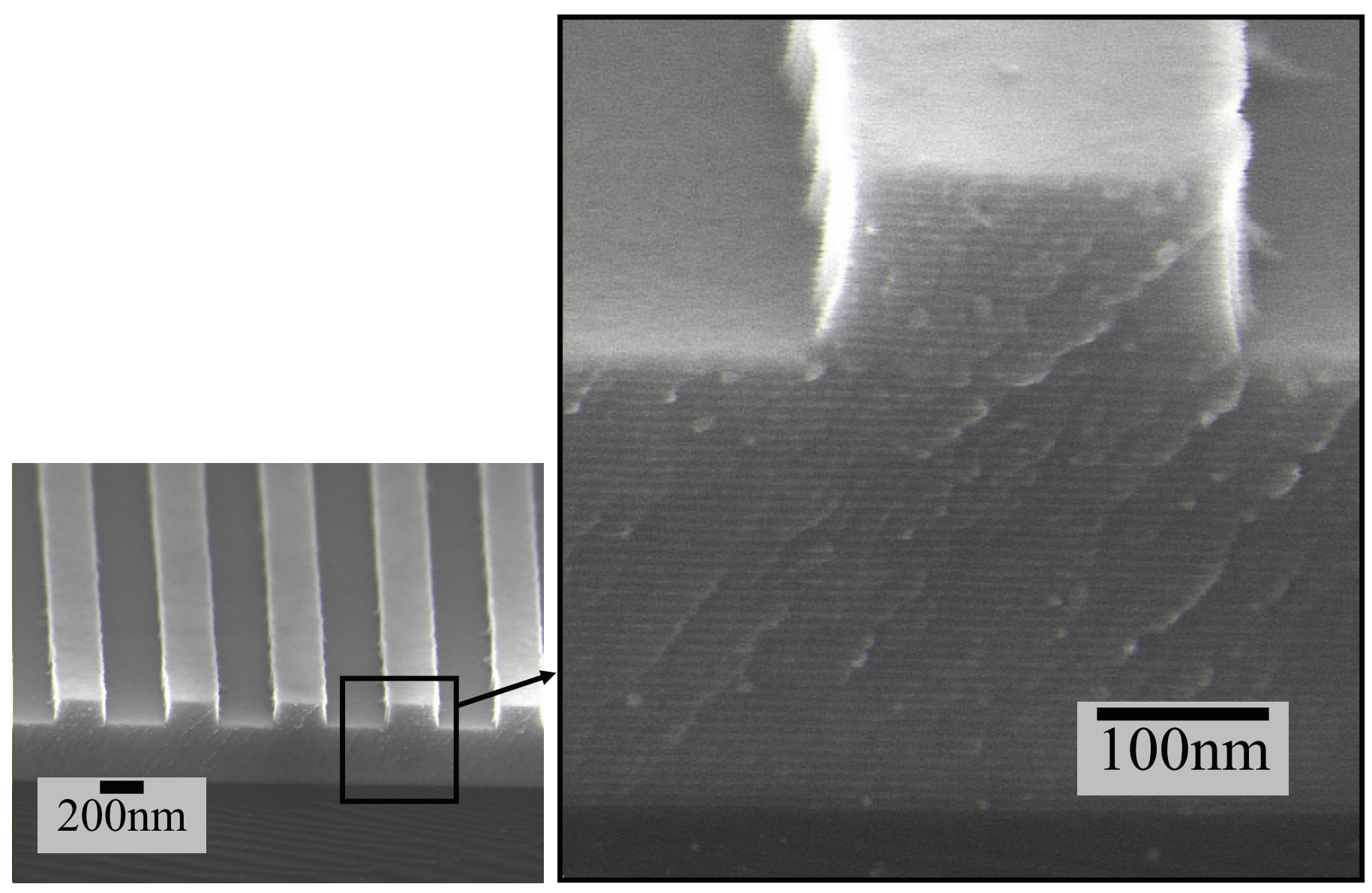


Figure 3

Grating efficiency as a function of duty cycle

a)

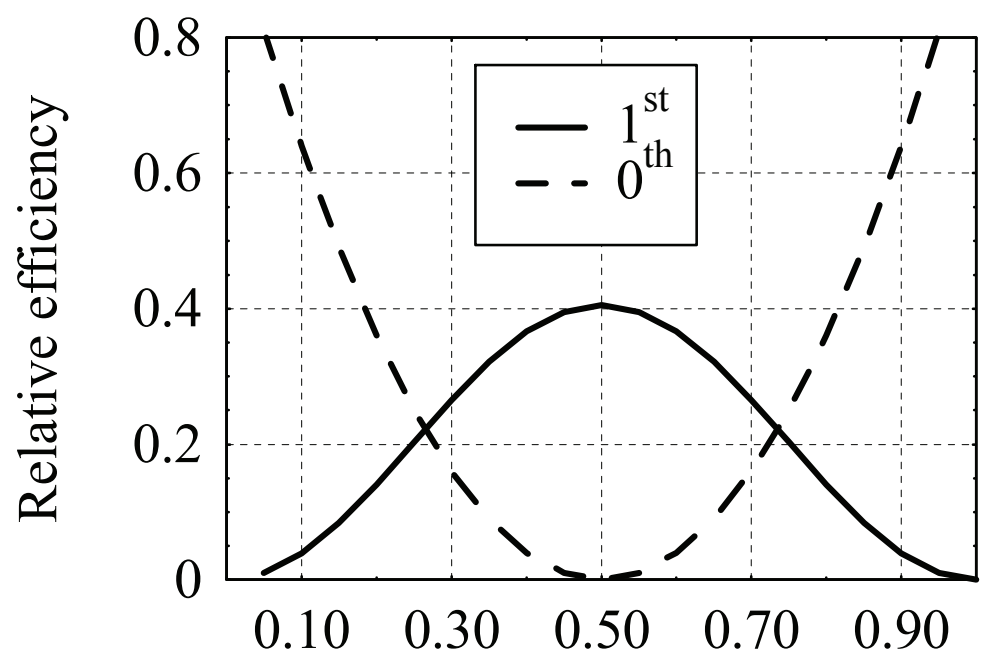

Gap/period ratio (duty cycle)

Grating efficiency as a function of phase

b)

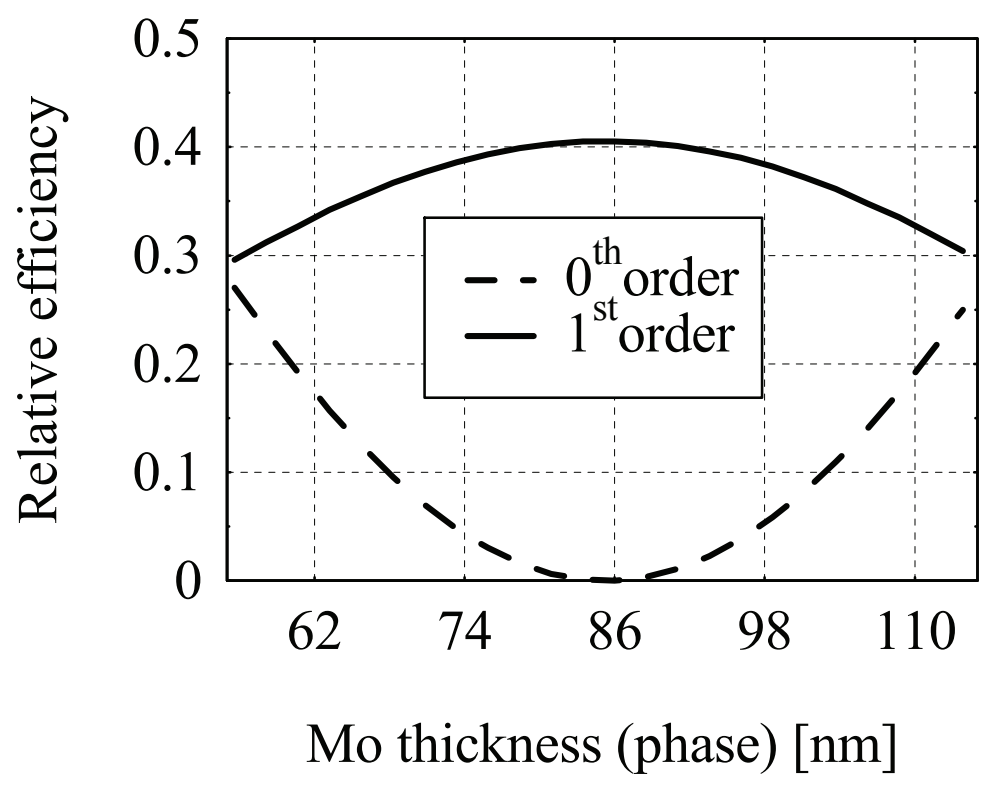


Figure 4

$\mathrm{Mo} / \mathrm{Si}$ reflectance with embedded $\mathrm{Cr}$ Structure: $(\mathrm{Si} / \mathrm{Mo})_{16} / \mathrm{Cr} /(\mathrm{Si} / \mathrm{Mo})_{40}$

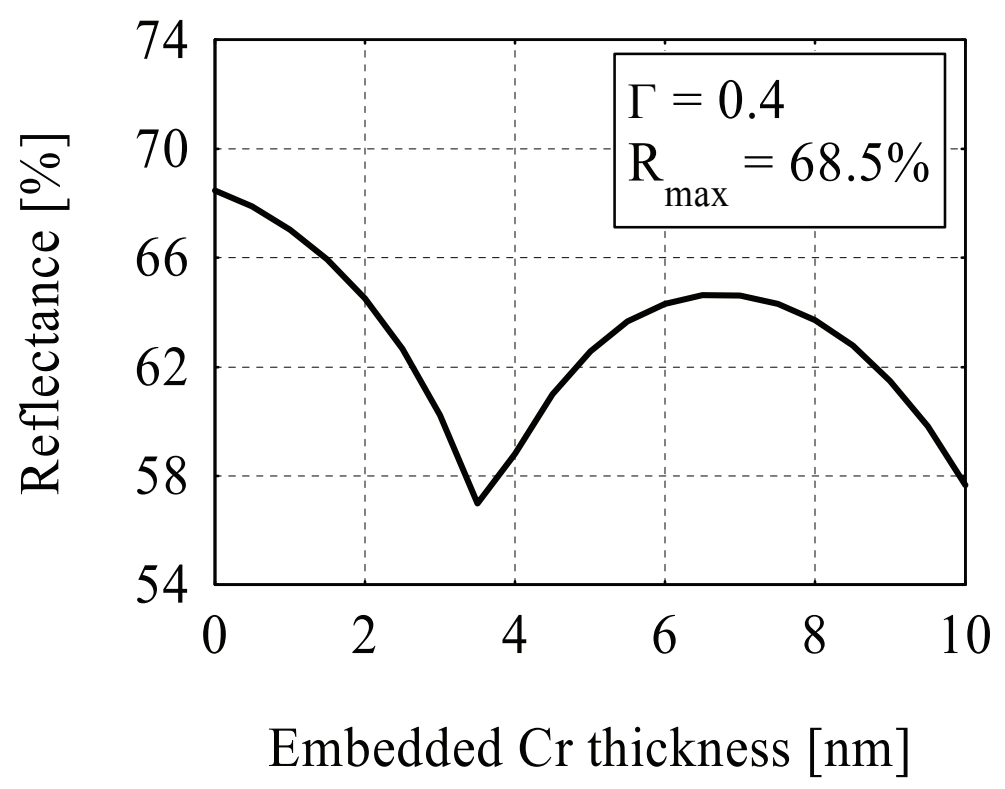


Figure 5
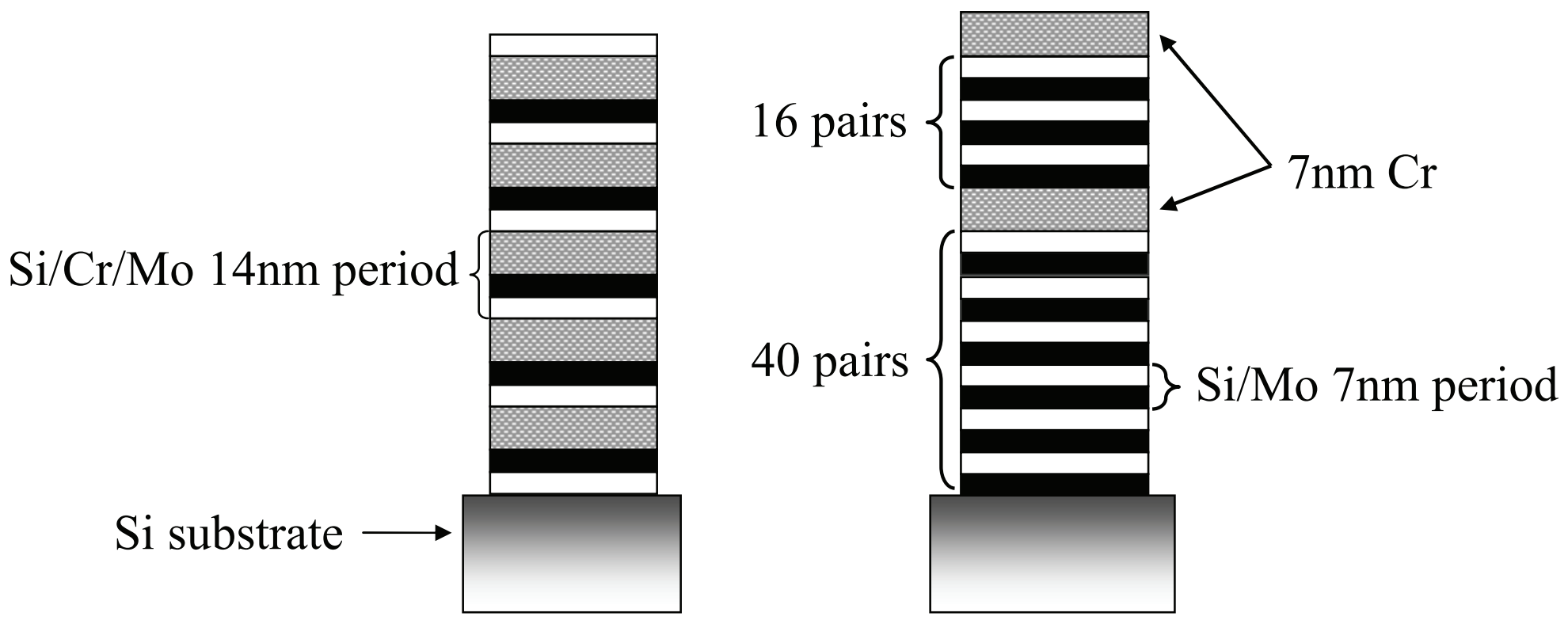

a)

b) 
Figure 6

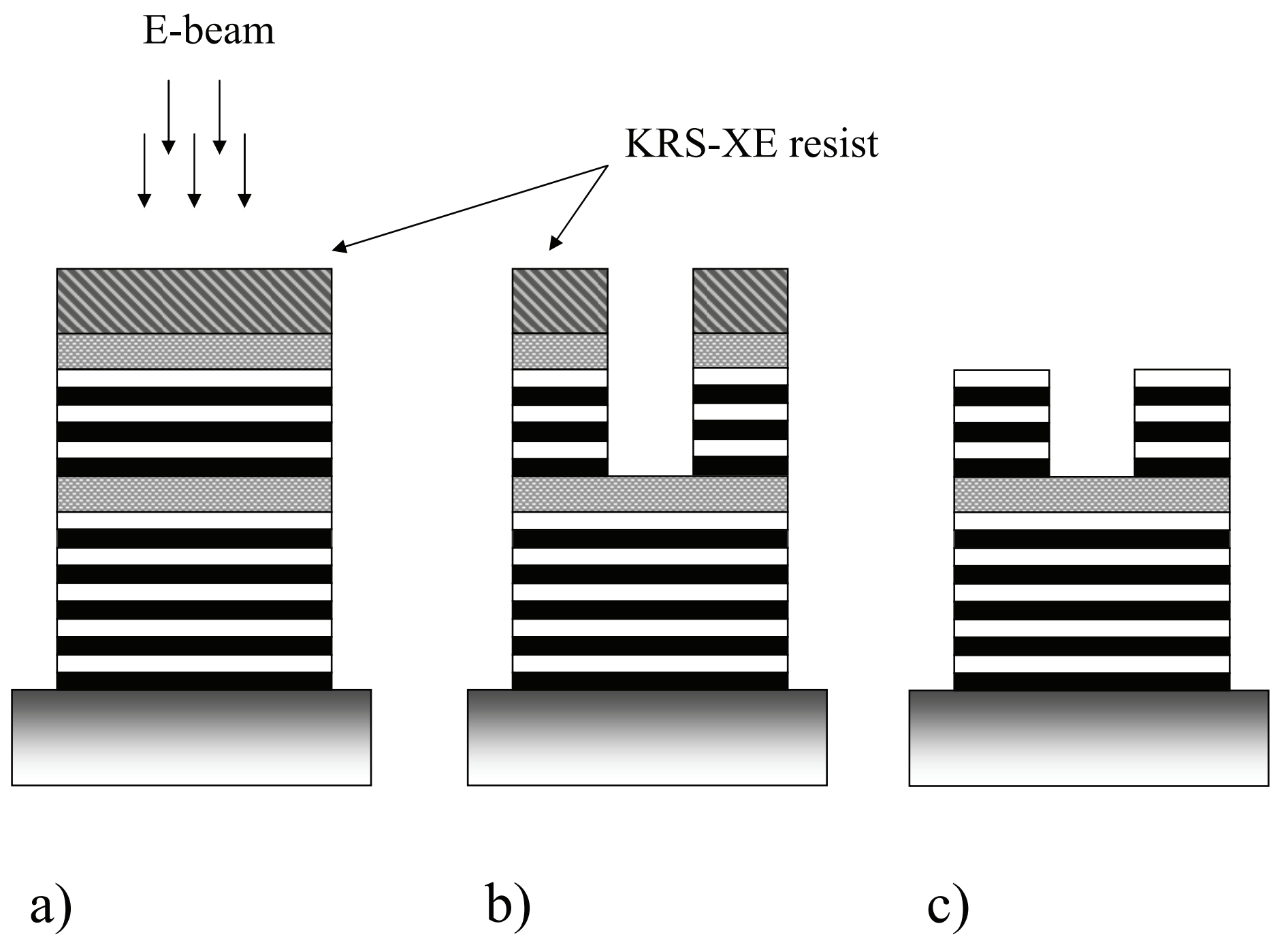


Grating efficiencies

a)

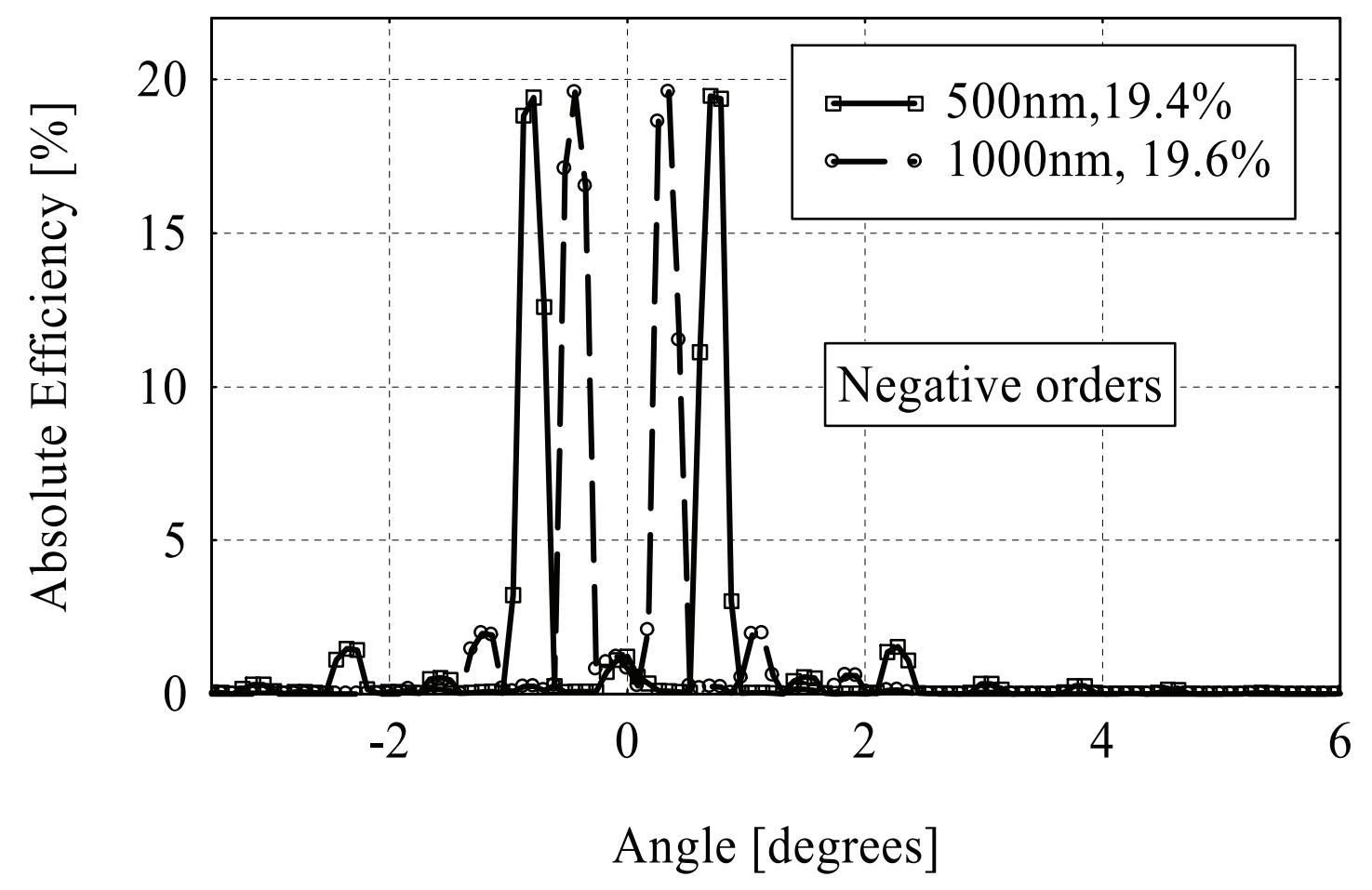

$\mathrm{Mo} / \mathrm{Si}$ with burried $\mathrm{Cr}$

b)

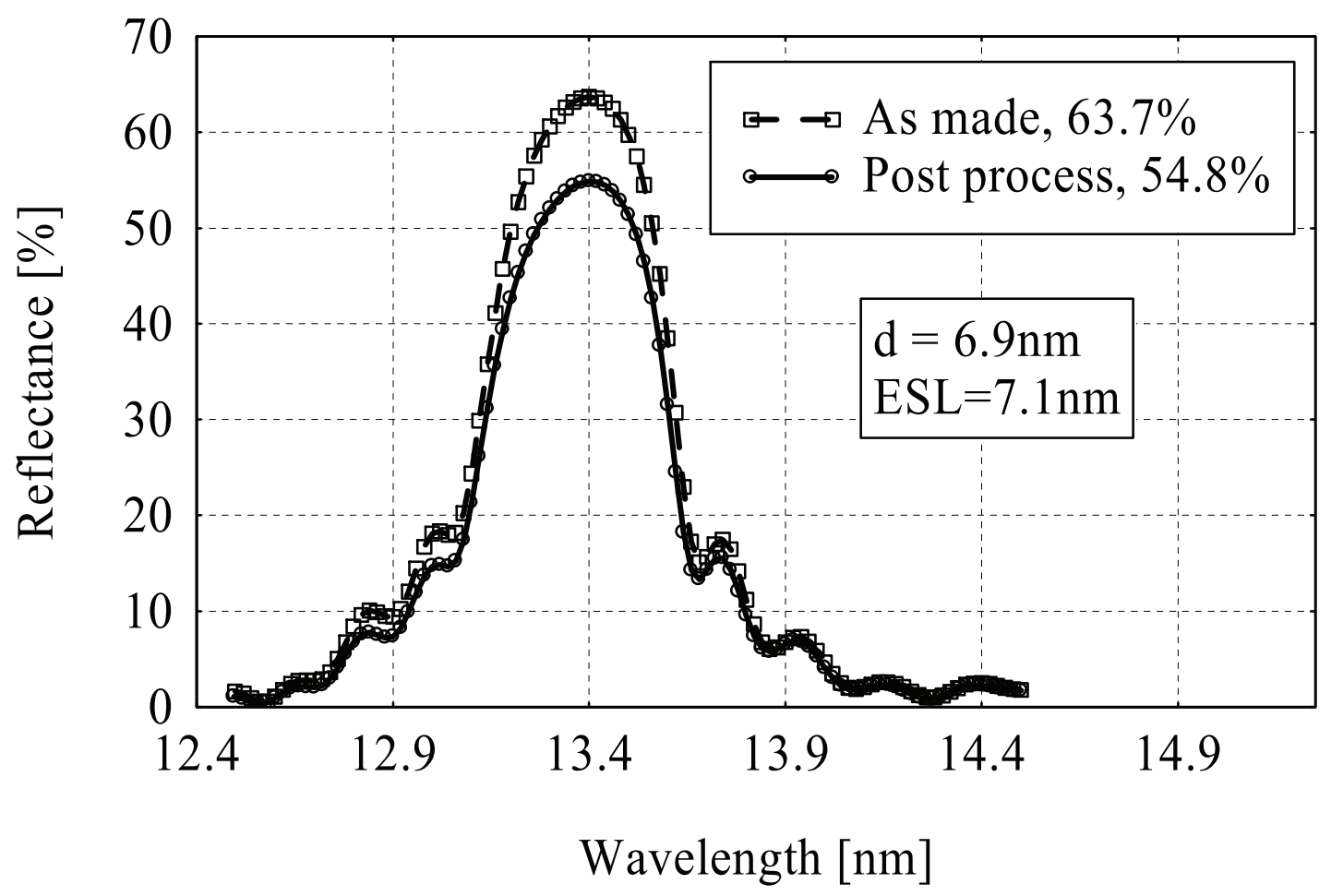




\section{References}

${ }^{1}$ F. Salmassi, P. Naulleau, E. Gullikson, D. Olynick, J. Liddle, "EUV Binary Phase Gratings: Fabrication and Application to Diffractive Optics," J. Vac. Sci. \& Technol. B, 24, 1136-1140 (2006).

${ }^{2}$ E.M. Gullikson, D. G. Stearns, “Asymmetric extreme ultraviolet scattering from sputter-deposited multilayers,” Phys Rev. B. 59, 13273 (1999).

${ }^{3}$ J.A. Liddle, F. Salmassi, P.P. Naulleau, E.M. Gullikson, "Nanoscale topography control for the fabrication of advanced diffracitve optics,'J. Vac. Sci. \& Technol. B, 21, 2980 (2003).

${ }^{4}$ P.P. Naulleau, J.A. Liddle, F. Salmassi, E.H. Anderson and E.M. Gullikson, "Design, fabrication, and characterization of high-efficiency extreme-ultraviolet diffusers."

Applied Optics, 43, 28, 1 (2004).

${ }^{5}$ Patrick P. Naulleau, James A. Liddle, Farhad Salmassi, Erik H. Anderson, and Eric M. Gullikson, "Design and fabrication of advanced EUV diffractive elements," Proc. SPIE. 5347, 9 (2004).

${ }^{6}$ P. Naulleau, C. Cho, E. Gullikson, and J. Bokor, "Transmission phase gratings for EUV interferometry,” J. Synch. Rad. 7, 405-410 (2000).

${ }^{7}$ Sang-In Han, E. Weisbrod, Qianghua Xie, P.J.S. Mangat, S.D. Hector, W.J. Dauksher, "Design and method of fabricating phase-shift masks for extreme-ultraviolet lithography by partial etching into the EUV multilayer mirror.” SPIE Proc. 5037, 314 (2003).

${ }^{8}$ Sang-In Han, E. Weisbrod, JR Wasson, R. Gregory, Qianghua Xie, PJS Mangat, SD Hector, WJ Dauksher, KM Rosfjord. "Development of phase shift masks for extreme ultra violet lithography and optical evaluation of phase shift materials." SPIE Proc. 5374, 261 (2004).

${ }^{9}$ P. P. Naulleau,J.A. Liddle, F. Salmassi, E. H. Anderson, E. M. Gullikson, "Design and fabrication of advanced EUV diffractive elements," Photonics West, San Jose, CA (US), 01/24/2004--01/29/2004 ; PBD: 16 Nov 2003

${ }^{10}$ Deirdre Olynick, Farhad Salmassi, J. Alex Liddle, to appear in J. Vac. Sci. \& Technol.

${ }^{11}$ Bajt S, Stearns DG, Kearney PA. "Investigation of the amorphous-to-crystalline transition in Mo/Si multilayers." Journal of Applied Physics, 90, 2 (2001).

${ }^{12}$ E. H. Anderson, V. Boegli, and L. P. Muray, " Electron Beam Lithography Digital Pattern Generator and Electronics for Generalized Curvilinear Structures," J. Vac.

Sci. Technol. B 13, 2529-2534 (1995). 
${ }^{13}$ E.M. Gullikson, S. Mrowka, B.B. Kaufmann, "Recent Developments in EUV Reflectometry at the Advanced Light Source," SPIE Proceedings 4343, 363 (2001) 